\title{
G-ANR's with homotopy trivial fixed point sets
}

\author{
by \\ Sergey A. Antonyan (México) \\ Dedicated to the memory of Professor Karol Borsuk \\ on the occasion of his centennial
}

\begin{abstract}
Let $G$ be a compact group and $X$ a $G$-ANR. Then $X$ is a $G$-AR iff the $H$-fixed point set $X^{H}$ is homotopy trivial for each closed subgroup $H \subset G$.
\end{abstract}

1. Introduction. The purpose of this paper is to prove the following

Main Theorem. Let $G$ be a compact group and $X$ a $G$-ANR. Then $X$ is a $G$-AR iff the $H$-fixed point set $X^{H}$ is homotopy trivial for each closed subgroup $H \subset G$.

This is the equivariant version of a well known result in the theory of retracts asserting that an ANR is an AR iff it is homotopy trivial [14, Theorem 4.2.20].

Only the "if" part of the Main Theorem is nontrivial. Since a G-ANR is a $G$-AR iff it is $G$-contractible (see [1, Theorem 6$]$ ), one just needs to establish the $G$-contractibility of $X$. For $G$ a compact Lie group this follows from the following James-Segal Theorem [10]: a $G$-map $f: X \rightarrow Y$ of $G$-ANR's is a $G$-homotopy equivalence if its restriction $f^{H}: X^{H} \rightarrow Y^{H}$ to the $H$-fixed point sets is an ordinary homotopy equivalence for every closed subgroup $H \subset G$. Validity of the Main Theorem for any compact (not necessarily Lie) group actions leads us to believe that the James-Segal Theorem should be true in this general case as well.

Our proof of the Main Theorem, even in the case of a compact Lie group $G$, relies neither on the James-Segal Theorem nor on its proof. We give a short proof based on the notion of an approximate slice (see Theorem 2.2 below) which is applicable at once to arbitrary compact group ac-

2000 Mathematics Subject Classification: 54C55, 55P91.

$K e y$ words and phrases: $G$-ANR, $H$-fixed point set, $G$-nerve, slice.

The author was supported by the grant U42563-F from CONACYT (México). 
tions. Another important ingredient in our proof is the Jaworowski-Lashof equivariant extension theorem [11], [13] (see Section 2).

The following example describes a situation where the Main Theorem applies in an essential way. Let $X$ be a Peano continuum (i.e., a connected, locally connected, compact metric space containing more than one point) on which a compact group $G$ acts nontransitively. Let $\exp X$ be the hyperspace of all nonempty compact subsets of $X$ endowed with the Hausdorff metric topology and the induced action of $G$. Set $Y=(\exp X) \backslash\{X\}$. We claim that $Y$ is a $G$-AR. Indeed, it is proved in [5, Proposition 3.1] that $\exp X$ is a $G$-AR, so $Y$ is a $G$-ANR. Hence, to prove that $Y$ is a $G$-AR, it suffices to show that $Y$ is $G$-contractible. However, there is no canonical way to contract $Y$ equivariantly to a $G$-fixed point of $Y$ (although $\exp X$ is canonically $G$ contractible to its $G$-fixed point $\{X\})$.

But the Main Theorem gives an answer here. Namely, if $H \subset G$ is a closed subgroup, then $(\exp X)^{H} \cong \exp (X / H)$. Since the $H$-orbit space $X / H$ is a Peano continuum (it is not a singleton because $G$ acts nontransitively on $X$ ) we infer that, due to the Curtis-Schori-West Hyperspace Theorem (see [14, Theorem 4.2.27]), $\exp (X / H)$ is a Hilbert cube. Consequently, $Y^{H}=(\exp X)^{H} \backslash\{X\}$ is a Hilbert cube with a removed point and hence is contractible. Now the Main Theorem works.

In connection with this example it is worth recalling that every compact metrizable (not necessarily Lie) group $G$ can act effectively (i.e., each $g \in G$ acts as a nontrivial homeomorphism) and nontransitively on a Peano continuum. What is more, such a group can even act effectively and nontransitively on the Hilbert cube. Indeed, according to a well-known result of Pontryagin [19, Ch. 8, Theorem 68], every compact metrizable group $G$ can be represented as the limit of an inverse system $\left\{G_{n}, \pi_{n, n+1}: n=1,2, \ldots\right\}$ of compact Lie groups $G_{n}$ and their continuous epimorphisms $\pi_{n, n+1}: G_{n+1} \rightarrow G_{n}$ such that each limit homomorphism $G \rightarrow G_{n}$ is also an epimorphism. Then for each $n \geq 1$ we consider $\operatorname{Cone}\left(G_{n}\right)$, the cone with base $G_{n}$. Since $G_{n}$ is a compact $\mathrm{ANR}$, Cone $\left(G_{n}\right)$ is a compact AR containing more than one point. Consequently, by a well-known result of West [20], $\prod_{n=1}^{\infty} \operatorname{Cone}\left(G_{n}\right)$ is homeomorphic to the Hilbert cube. On the other hand, since $G_{n}$ is the quotient group $G / H_{n}$ for a closed normal subgroup $H_{n} \subset G$, we see that $G$ acts naturally (on the left) on $G_{n}$, and this action extends to an action of $G$ on Cone $\left(G_{n}\right)$. Next, since $\bigcap_{n=1}^{\infty} H_{n}$ only contains the unity of $G$, the induced diagonal action of $G$ on the Hilbert cube $\prod_{n=1}^{\infty}$ Cone $\left(G_{n}\right)$ is effective. To complete our construction it remains to observe that this action has a (unique) $G$-fixed point, and hence, is nontransitive. By the way, no compact group can act transitively on the Hilbert cube; we leave the details of the proof to the reader as a stimulating exercise. 
It is also interesting to mention that $\prod_{n=1}^{\infty} \operatorname{Cone}\left(G_{n}\right)$ is, in fact, a $G$-AR. This is because each coset $G_{n}=G / H_{n}$, being a Lie group, is a $G$-ANR (see Proposition 2.3 below). In turn, this implies that Cone $\left(G_{n}\right), n \geq 1$, is a $G$-AR (see [4, Proposition 2.2]), and hence $\prod_{n=1}^{\infty} \operatorname{Cone}\left(G_{n}\right)$ is also a $G$-AR. Thus, to each representation of a compact metrizable group $G$ as the limit of an inverse system of compact Lie groups corresponds an effective (and nontransitive) $G$-action on the Hilbert cube, making the latter a $G$-AR.

Perhaps it is in order to recall here yet another (and more universal) source of $G$-ANR's: these are the mapping spaces. Namely, it is proved in [2, Theorem 8] that for any compact group $G$ and any $\mathrm{A}(\mathrm{N}) \mathrm{R}$ space $L$, the space $C(G, L)$ of all continuous mappings $f: G \rightarrow L$, endowed with the compact-open topology, is a $G$-A(N)R. Here $G$ acts on $C(G, L)$ according to the rule $(g, f) \mapsto g f$, where $(g f)(t)=f(t g), t \in G$. In particular, if $L$ is a normed linear space then $C(G, L)$ is a $G$-AR. Moreover, each $G-\mathrm{A}(\mathrm{N}) \mathrm{R}$ is a (neighborhood) $G$-retract of $C(G, L)$ for a suitably chosen normed linear space $L$ [2, Corollary 5].

We conclude this introduction by considering yet another example where the Main Theorem substantially helps to establish that a given $G$-ANR space is, in fact, a $G$-AR. Let $G$ be any compact group and $L$ an infinitedimensional normed linear space. Further, let $X$ denote the above mentioned normed linear $G$-space $C(G, L)$ with the origin removed. Since $C(G, L)$ is a $G$-AR we see that $X$ is a $G$-ANR. But in fact $X$ is a $G$-AR, though, after removing the origin, the $G$-contractibility of $X$ is not evident. First we observe that for any closed subgroup $H \subset G, X^{H}=C(G, L)^{H} \backslash\{0\}$. Further, it is easy to see that $C(G, L)^{H}$ is homeomorphic to $C(G / H, L)$, where $G / H$ denotes the right coset space. Since $C(G / H, L)$ is an infinite-dimensional normed linear space, according to a result of Klee [12], the complement $X^{H}=C(G / H, L) \backslash\{0\}$ is homeomorphic to $C(G / H, L)$. It then follows that $X^{H}$ is contractible, and hence homotopy trivial. It remains to apply the Main Theorem.

Finally, we refer the reader to [4] where the "Lie case" of the Main Theorem is applied in the study of proper actions (in the sense of R. Palais [18]) of noncompact Lie groups. We hope that availability of the Main Theorem for non-Lie groups will now foster further development of the theory of proper actions of arbitrary locally compact groups.

2. Preliminaries. Throughout the paper the letter $G$ will denote a compact Hausdorff topological group unless otherwise stated; by $e$ we denote the unit element of $G$.

The basic ideas and facts of the theory of $G$-spaces or topological transformation groups can be found in Bredon [7] and in Palais [17]. For the equivariant theory of retracts the reader can see, for instance, the papers 
[1], [2] and [6]. Below, for the convenience of the reader, we recall some more special definitions and facts.

All spaces are assumed to be completely regular and Hausdorff. All $G$ maps are assumed to be continuous.

A space $X$ is called homotopy trivial if for every $n \geq 0$, every continuous map from the sphere $S^{n}$ to $X$ can be continuously extended over the closed ball $B^{n+1}$.

A metrizable $G$-space $Y$ is called a $G$-equivariant absolute neighborhood retract (for the class of all metrizable $G$-spaces) (notation: $Y \in G$-ANR) if, for any closed invariant subset $A$ of a metrizable $G$-space $X$ and any $G$-map $f: A \rightarrow Y$, there exist an invariant neighborhood $U$ of $A$ in $X$ and a $G$-map $\psi: U \rightarrow Y$ that extends $f$. If, in addition, one can always take $U=X$, then we say that $Y$ is a $G$-equivariant absolute retract (notation: $Y \in G$-AR). The map $\psi$ is called a $G$-extension of $f$.

For a point $x$ of a $G$-space $X$, the subgroup $G_{x}=\{g \in G \mid g x=x\}$ is called the stabilizer or isotropy subgroup of $x$. For a subgroup $H \subset G$, the set $X^{H}=\left\{x \in X \mid H \subset G_{x}\right\}$ is called the $H$-fixed point set of $X$. We denote by $G / H$ the $G$-space of cosets $\{g H \mid g \in G\}$ under the action induced by left translations.

The family $(H)=\left\{g H g^{-1} \mid g \in G\right\}$ of all subgroups of $G$ which are conjugate to a given subgroup $H \subset G$ is called a $G$-orbit type. One says that a $G$-space $X$ has finitely many $G$-orbit types if there exist a finite number of closed subgroups $H_{1}, \ldots, H_{n}$ of $G$ such that $\left(G_{x}\right) \in\left\{\left(H_{1}\right), \ldots,\left(H_{n}\right)\right\}$ for all $x \in X$.

If $X$ is a $G$-space and $S \subset X$ then the set $G(S)=\{g s \mid g \in G, s \in S\}$ is called the saturation of $S$.

The notion of a slice is the key tool in our proofs, so let us recall it:

Definition 2.1 ([17]). Let $X$ be a $G$-space and $H \subset G$ a closed subgroup. A subset $S \subset X$ is called an $H$-slice in $X$ if:

(1) $S$ is $H$-invariant,

(2) the saturation $G(S)$ is open in $X$,

(3) if $g \in G \backslash H$, then $g S \cap S=\emptyset$,

(4) $S$ is closed in $G(S)$.

The saturation $G(S)$ is called an $H$-tube, and $H$ is called a slicing subgroup.

Each $H$-slice $S$ uniquely determines a $G$-map $f: G(S) \rightarrow G / H$ such that $f^{-1}(e H)=S$ [17, Theorem 1.7.7]. We will call such a $G$-map $f$ the slicing map.

The classical Slice Theorem, which in its final form was proved in Mostow [16], asserts that if $G$ is a compact Lie group and $x$ any point of a given $G$ space $X$, then there exists a $G_{x}$-slice $S \subset X$ that contains $x$. This theorem is 
no longer true if the acting group is not Lie (see [3]). However, the following approximate version of the Slice Theorem remains true for arbitrary compact group actions:

Theorem 2.2 (Approximate Slice Theorem [3]). Let $X$ be a $G$-space and $x \in X$. Then for any neighborhood $U$ of $x$ in $X$, there exist a large subgroup $K \subset G$ with $G_{x} \subset K$ and a $K$-slice $S$ such that $x \in S \subset U$.

Here a closed subgroup $K$ of a compact group is called large [3] if there exists a closed normal subgroup $N$ of $G$ such that $N \subset K$ and $G / N$ is a Lie group. Large subgroups are characterized by the following proposition:

Proposition 2.3 ([3]). For a closed subgroup $H \subset G$, the following conditions are equivalent:

(1) $H$ is a large subgroup,

(2) $G / H$ is a $G-A N R$,

(3) $G / H$ is locally contractible,

(4) $G / H$ is a smooth manifold.

Let $X$ be a $G$-space, $H \subset G$ a large subgroup, $S$ an $H$-slice in $X$, and $O \subset G$ a neighborhood of the identity. The set $g O S=\{g p s \mid p \in O, s \in S\}$, where $g \in G$, is called a tubular segment of type $H$.

A family

$$
\mathcal{U}=\left\{g O_{\mu} S_{\mu} \mid g \in G, \mu \in \mathcal{M}\right\}
$$

consisting of tubular segments with large slicing subgroups $\left\{H_{\mu} \mid \mu \in \mathcal{M}\right\}$ is called a $G$-normal cover of $X$ if the family $\widetilde{\mathcal{U}}=\left\{G\left(S_{\mu}\right) \mid \mu \in \mathcal{M}\right\}$ of open tubes covers $X$ and there exists an invariant locally finite partition of unity $\left\{\varphi_{\mu}: X \rightarrow[0,1] \mid \mu \in \mathcal{M}\right\}$ subordinated to $\widetilde{\mathcal{U}}$, i.e., every $\varphi_{\mu}$ is an invariant function with $\varphi_{\mu}^{-1}((0,1]) \subset G\left(S_{\mu}\right)$.

If $X$ is paracompact then the orbit space $X / G$, being a closed image of $X$, is paracompact as well (see [9, Ch. VIII, Theorem 2.4]). In this case each cover of the form $\mathcal{U}=\left\{g O_{\mu} S_{\mu} \mid g \in G, \mu \in \mathcal{M}\right\}$, where $S_{\mu}$ is an $H_{\mu}$-slice with a large slicing subgroup $H_{\mu}$, is a $G$-normal cover.

The following result is based on the Approximate Slice Theorem 2.2 and plays an important role in this paper:

Lemma $2.4([6])$. Let $X$ be a paracompact $G$-space. Then for each open cover $\mathcal{V}$ of $X$ there exists a $G$-normal cover $\mathcal{U}=\left\{g O_{\mu} S_{\mu} \mid g \in G, \mu \in \mathcal{M}\right\}$ of $X$ with large slicing subgroups $\left\{H_{\mu} \mid \mu \in \mathcal{M}\right\}$ such that $\mathcal{U}$ is a refinement of $\mathcal{V}$.

Definition 2.5. Let $X$ be a $G$-space, $U$ an open invariant subset of $X$ and $\mathcal{U}=\left\{g O_{\mu} S_{\mu} \mid g \in G, \mu \in \mathcal{M}\right\}$ a $G$-normal cover of $U$. Then $\mathcal{U}$ is called a Dugundji $G$-cover of $U$ (with respect to $X$ ) if for any point $a \in X \backslash U$ and 
any neighborhood $V_{a}$ of $a$ in $X$, there exists a neighborhood $W_{a} \subset V_{a}$ of $a$ in $X$ such that every $g O_{\mu} S_{\mu}$ which meets $W_{a}$ is contained in $V_{a}$.

Lemma 2.6. If $X$ is a metrizable $G$-space, then every invariant open subset $U \subset X$ admits a Dugundji $G$-cover with respect to $X$.

Proof. If $U=X$, then the one-element cover $\{X\}$ (with $G$ as the slicing subgroup) is a Dugundji $G$-cover of $U$.

Let $U \neq X$. We consider an invariant metric $\varrho$ on $X$ (see, e.g., [17, Proposition 1.1.12]). In what follows we shall denote by $N(x, r)$ the open ball in $X$ of radius $r$ centered at $x \in X$. Let $r_{x}=(1 / 4) \varrho(x, X \backslash U)$. Consider the index set $\mathcal{M}=U / G$. In each orbit $\mu \in \mathcal{M}$ we choose a point $x_{\mu}$; so $\mu=G\left(x_{\mu}\right)$. By continuity of the $G$-action on $X$, choose a neighborhood $O_{\mu}$ of the unity in $G$ and a number $0<\delta_{\mu}<r_{x_{\mu}}$ such that $O_{\mu} N\left(x_{\mu}, \delta_{\mu}\right) \subset$ $N\left(x_{\mu}, r_{x_{\mu}}\right)$ (recall that for $F \subset G$ and $A \subset X$ we denote by $F A$ the set $\{g a \mid g \in F, a \in A\})$.

By Theorem 2.2, there exist a large subgroup $H_{\mu}$ of $G$ with $G_{x_{\mu}} \subset H_{\mu}$ and an $H_{\mu}$-slice $S_{\mu}$ such that $x_{\mu} \in S_{\mu} \subset N\left(x_{\mu}, \delta_{\mu}\right)$. Clearly, $O_{\mu} S_{\mu} \subset N\left(x_{\mu}, r_{x_{\mu}}\right)$.

We claim that the $G$-normal cover $\left.\mathcal{U}=\left\{g O_{\mu} S_{\mu}\right\} \mid g \in G, \mu \in \mathcal{M}\right\}$ is a Dugundji $G$-cover of $U$ with large slicing subgroups $\left\{H_{\mu} \mid \mu \in \mathcal{M}\right\}$. Indeed, since the orbit space $U / G$ is metrizable (see [17, Proposition 1.1.12]), and hence paracompact, there exists an invariant partition of unity subordinated to the cover $\left\{G\left(S_{\mu}\right) \mid \mu \in \mathcal{M}\right\}$.

Next, let $a \in X \backslash U$ and $V_{a}$ a neighborhood of $a$. Choose $\varepsilon>0$ such that $N(a, 2 \varepsilon) \subset V_{a}$. We will show that $W_{a}=N(a, \varepsilon / 2)$ is the desired neighborhood of $a$. In fact, let $g O_{\mu} S_{\mu} \cap W_{a} \neq \emptyset$, and let $y \in g O_{\mu} S_{\mu} \cap W_{a}$. Since $y \in W_{a}$, one has $\varrho(a, y)<\varepsilon / 2$. On the other hand, $y \in g O_{\mu} S_{\mu}$, implying

$$
\varrho\left(y, g x_{\mu}\right) \leq \operatorname{diam} g O_{\mu} S_{x_{\mu}} .
$$

Since $O_{\mu} S_{\mu} \subset N\left(x_{\mu}, r_{x_{\mu}}\right)$, we see that $\operatorname{diam} O_{\mu} S_{\mu} \leq 2 r_{x_{\mu}}$. Now, by the invariance of the metric $\varrho$, we have

$$
\begin{aligned}
\operatorname{diam} g O_{\mu} S_{\mu}=\operatorname{diam} O_{\mu} S_{\mu} \leq 2 r_{x_{\mu}} & =(1 / 2) \varrho\left(x_{\mu}, X \backslash U\right) \\
& =(1 / 2) \varrho\left(g x_{\mu}, X \backslash U\right) .
\end{aligned}
$$

Since $a \in X \backslash U$, one has $\varrho\left(g x_{\mu}, X \backslash U\right) \leq \varrho\left(g x_{\mu}, a\right)$. Consequently, (2.1) and (2.2) yield

$$
\varrho\left(y, g x_{\mu}\right) \leq(1 / 2) \varrho\left(g x_{\mu}, a\right) .
$$

Using this inequality and the triangle inequality, we get

$$
\varrho\left(a, g x_{\mu}\right) \leq \varrho(a, y)+\varrho\left(y, g x_{\mu}\right)<\varepsilon / 2+(1 / 2) \varrho\left(a, g x_{\mu}\right) .
$$

This yields $\varrho\left(a, g x_{\mu}\right)<\varepsilon$, which together with $(2.2)$ implies that

$$
\operatorname{diam} g O_{\mu} S_{\mu} \leq(1 / 2) \varrho\left(g x_{\mu}, X \backslash U\right) \leq(1 / 2) \varrho\left(g x_{\mu}, a\right)<\varepsilon / 2 .
$$


Consequently, since $g O_{\mu} S_{\mu}$ meets $W_{a}=N(a, \varepsilon / 2)$, it follows from the inequality diam $g O_{\mu} S_{\mu}<\varepsilon / 2$ that $g O_{\mu} S_{\mu} \subset N(a, 2 \varepsilon)$. Since $N(a, 2 \varepsilon) \subset V_{a}$, the proof is finished.

We shall need the following theorem which is a particular case of a result proved in Jaworowski [11] and Lashof [13]:

THEOREM 2.7 (Jaworowski-Lashof). Let $G$ be a compact Lie group, $X$ a finite-dimensional, separable, metrizable $G$-space with a finite number of $G$-orbit types, and $A$ a closed invariant subset of $X$. Suppose $f: A \rightarrow Y$ is a G-map into a metrizable $G$-space $Y$. If the $H$-fixed point set $Y^{H}$ is an $A R$ for each closed subgroup $H \subset G$, then $f$ extends to a $G$-map $F: X \rightarrow Y$.

3. Replacement by a $G$-nerve. First we recall the definition of the $G$-nerve of a $G$-normal cover [6].

Let $X$ be a $G$-space and $\mathcal{U}=\left\{g O_{\mu} S_{\mu} \mid g \in G, \mu \in \mathcal{M}\right\}$ a $G$-normal cover of $X$ with large slicing subgroups $\left\{H_{\mu} \mid \mu \in \mathcal{M}\right\}$. Let $\tilde{\mathcal{N}}(\mathcal{U})$ be the ordinary nerve of the invariant cover $\left\{G\left(S_{\mu}\right) \mid \mu \in \mathcal{M}\right\}$. We shall denote by $\left\langle\mu_{0}, \ldots, \mu_{n}\right\rangle$ the closed $n$-simplex of $\tilde{\mathcal{N}}(\mathcal{U})$ corresponding to the sets $G\left(S_{\mu_{0}}\right), \ldots, G\left(S_{\mu_{n}}\right)$ with $G\left(S_{\mu_{0}}\right) \cap \cdots \cap G\left(S_{\mu_{n}}\right) \neq \emptyset$. Let $f_{\mu}: G\left(S_{\mu}\right) \rightarrow$ $G / H_{\mu}$ be the corresponding slicing map (see Section 2). For any simplex $\sigma=\left\langle\mu_{0}, \ldots, \mu_{n}\right\rangle \subset \widetilde{\mathcal{N}}(\mathcal{U})$, we define the following subset of the product $\prod_{i=0}^{n} G / H_{\mu_{i}}$ endowed with the diagonal $G$-action:

$$
F_{\sigma}=\left\{\left(f_{\mu_{0}}(x), \ldots, f_{\mu_{n}}(x)\right) \mid x \in \bigcap_{i=0}^{n} G\left(S_{\mu_{i}}\right)\right\} \text {. }
$$

It follows from the equivariance of $f_{\mu_{i}}$ that $F_{\sigma}$ is an invariant subset of the $G$ space $\prod_{i=0}^{n} G / H_{\mu_{i}}$. Observe that if $\tau$ is a subsimplex of $\sigma$, then $q_{\sigma \tau}\left(F_{\sigma}\right) \subset F_{\tau}$, where $q_{\sigma \tau}: \prod_{\mu \in \sigma} G / H_{\mu} \rightarrow \prod_{\mu \in \tau} G / H_{\mu}$ is the Cartesian projection.

For a simplex $\sigma=\left\langle\mu_{0}, \ldots, \mu_{n}\right\rangle \subset \tilde{\mathcal{N}}(\mathcal{U})$, we denote by $\mathcal{J}(\sigma)$ the finite join

$$
G / H_{\mu_{0}} * \cdots * G / H_{\mu_{n}}
$$

in the sense of Milnor [15], equipped with the natural action of $G$. We shall use the notation $\sum_{i=0}^{n} t_{\mu_{i}} g_{\mu_{i}} H_{\mu_{i}}$ for the point in $\mathcal{J}(\sigma)$ determined by the elements $g_{\mu_{i}} H_{\mu_{i}} \in G_{\mu_{i}} / H_{\mu_{i}}$ and the numbers $t_{\mu_{i}} \geq 0$ with $\sum_{i=0}^{n} t_{\mu_{i}}=1$. The $t_{\mu_{i}}$ are called the barycentric coordinates of $\sum_{i=0}^{n} t_{\mu_{i}} g_{\mu_{i}} H_{\mu_{i}}$.

Since each $H_{\mu}$ is a large subgroup, $G / H_{\mu}$ is a compact metrizable $G$ space (see Proposition 2.3). This implies that $\mathcal{J}(\sigma)$ is a compact metrizable $G$-space as well.

Further, we denote by $\Delta\left(\sigma, F_{\sigma}\right)$ the invariant subset of $\mathcal{J}(\sigma)$ consisting of all those $\sum_{i=0}^{n} t_{\mu_{i}} g_{\mu_{i}} p_{\mu_{i}} H_{\mu_{i}} \in \mathcal{J}(\sigma)$ for which

$$
\left(g_{\mu_{0}} H_{\mu_{0}}, \ldots, g_{\mu_{n}} H_{\mu_{n}}\right) \in F_{\sigma} \quad \text { and } \quad p_{\mu_{i}} \in O_{\mu_{i}}, \quad 1 \leq i \leq n \text {. }
$$


Set

$$
\Delta(\sigma)=\bigcup\left\{\Delta\left(\tau, F_{\tau}\right) \mid \tau \text { is a subsimplex of } \sigma\right\} .
$$

Then $\Delta(\sigma)$ is a $G$-invariant subset of $\mathcal{J}(\sigma)$. We will consider the induced topology and $G$-action on $\Delta(\sigma)$.

We call $\Delta(\sigma)$ a closed $G$-n-simplex over the $n$-simplex $\sigma$ associated with the families

$$
\left\{H_{\mu_{0}}, \ldots, H_{\mu_{n}}\right\}, \quad\left\{O_{\mu_{0}}, \ldots, O_{\mu_{n}}\right\} \quad \text { and } \quad\left\{F_{\tau} \mid \tau \text { is a subsimplex of } \sigma\right\} .
$$

The set

$$
\partial \Delta(\sigma)=\Delta(\sigma) \backslash\left\{\sum_{i=0}^{n} t_{\mu_{i}} g_{\mu_{i}} H_{\mu_{i}} \in \Delta(\sigma) \mid t_{\mu_{i}}>0,0 \leq i \leq n\right\}
$$

is called the $G$-boundary of $\Delta(\sigma)$. The homogeneous spaces $G / H_{\mu_{0}}, \ldots, G / H_{\mu_{n}}$ are called the $G$-vertices of the $G$-simplex $\Delta(\sigma)$.

Next, if $g_{\mu_{0}} H_{\mu_{0}} \in G / H_{\mu_{0}}, \ldots, g_{\mu_{n}} H_{\mu_{n}} \in G / H_{\mu_{n}}$ are fixed elements, then the closed (resp., open) $n$-cell $\left\langle g_{\mu_{0}} H_{\mu_{0}}, \ldots, g_{\mu_{n}} H_{\mu_{n}}\right\rangle$ is defined to be the subset of $\mathcal{J}(\sigma)$ consisting of all $\sum_{i=0}^{n} t_{\mu_{i}} g_{\mu_{i}} H_{\mu_{i}}$, where $t_{i} \geq 0$ (res., $\left.t_{i}>0\right)$ and $\sum_{i=0}^{n} t_{\mu_{i}}=1$. The corresponding open $n$-cell is denoted by $\left(g_{\mu_{0}} H_{\mu_{0}}, \ldots, g_{\mu_{n}} H_{\mu_{n}}\right)$.

Consider the union

$$
\mathcal{N}(\mathcal{U})=\bigcup\{\Delta(\sigma) \mid \sigma \text { is a simplex of } \tilde{\mathcal{N}}(\mathcal{U})\}
$$

endowed with the weak topology determined by the family $\{\Delta(\sigma) \mid \sigma \subset$ $\tilde{\mathcal{N}}(\mathcal{U})\}$, i.e., a set $U \subset \mathcal{N}(\mathcal{U})$ is open in $\mathcal{N}(\mathcal{U})$ iff $U \cap \Delta(\sigma)$ is open in $\Delta(\sigma)$ for every simplex $\sigma \subset \tilde{\mathcal{N}}(\mathcal{U})$. If $\sigma$ and $\tau$ are two simplices of $\tilde{\mathcal{N}}(\mathcal{U})$, then it is easy to see that $\Delta(\sigma) \cap \Delta(\tau)=\Delta(\sigma \cap \tau)$. This shows that $\Delta(\sigma) \cap \Delta(\tau)$ is closed in both $\Delta(\sigma)$ and $\Delta(\tau)$, implying that each $G$-simplex $\Delta(\sigma)$ retains its original topology and is a closed subset of $\mathcal{N}(\mathcal{U})$ (see, e.g., [9, Ch. VI, $\S 8]$ ).

It is an easy exercise to show that the induced $G$-action on $\mathcal{N}(\mathcal{U})$ is continuous; thus $\mathcal{N}(\mathcal{U})$ is a $G$-space.

Definition $3.1([6])$. The $G$-space $\mathcal{N}(\mathcal{U})$, endowed with the weak topology and the $G$-action determined by the family of its $G$-simplices

$$
\{\Delta(\sigma) \mid \sigma \text { is a simplex of } \tilde{\mathcal{N}}(\mathcal{U})\},
$$

is called the equivariant nerve or $G$-nerve of the $G$-normal cover $\mathcal{U}$.

In what follows we shall use the easily checked fact that a map $f$ : $\mathcal{N}(\mathcal{U}) \rightarrow Z$ is continuous iff its restriction $\left.f\right|_{\Delta(\sigma)}$ to every closed $G$-simplex $\Delta(\sigma)$ is continuous.

Lemma $3.2([6])$. Let $Y$ be a $G$-space and $\mathcal{U}=\left\{g O_{\mu} S_{\mu} \mid g \in G, \mu \in \mathcal{M}\right\}$ a G-normal cover of $Y$. Then for each invariant partition of unity subordinated to the invariant cover $\left\{G\left(S_{\mu}\right) \mid \mu \in \mathcal{M}\right\}$, there exists a $G$-map 
$p: Y \rightarrow \mathcal{N}(\mathcal{U})$ such that the $\mu$ th barycentric coordinate of $p(y)$ vanishes whenever $y \in Y \backslash G\left(S_{\mu}\right)$.

Generalizing an idea of Dugundji [8], we define the replacement by a $G$-nerve.

Let $Y$ be a $G$-space, $A$ an invariant closed subset of $Y$, and let

$$
\mathcal{U}=\left\{g O_{\mu} S_{\mu} \mid g \in G, \mu \in \mathcal{M}\right\}
$$

be a $G$-normal cover of $Y \backslash A$ with large slicing subgroups $\left\{H_{\mu} \mid \mu \in \mathcal{M}\right\}$. Let $\mathcal{N}(\mathcal{U})$ be the $G$-nerve of $\mathcal{U}$ and let $D(\mathcal{U})$ denote the disjoint union $A \cup \mathcal{N}(\mathcal{U})$. Topologize $D(\mathcal{U})$ as follows. Let $z \in D(\mathcal{U})$ be an arbitrary point. If $z \in \mathcal{N}(\mathcal{U})$, a basis of neighborhoods of $z$ in $D(\mathcal{U})$ is taken to be all neighborhoods of $z$ in $\mathcal{N}(\mathcal{U})$. If $z \in A$, we define a basis of neighborhoods of $z$ in $D(\mathcal{U})$ to be all sets $\widetilde{W}$ defined as follows. For any neighborhood $W$ of $z$ in $Y$, let $\widetilde{W} \subset A \cup \mathcal{N}(\mathcal{U})$ be the set $A \cap W$ together with each open cell $\left(g_{1} p_{\mu_{1}} H_{\mu_{1}}, \ldots, g_{n} p_{\mu_{n}} H_{\mu_{n}}\right)$ in $\mathcal{N}(\mathcal{U}), g_{i} \in G, p_{\mu_{i}} \in O_{\mu_{i}}, 1 \leq i \leq n$, such that $g_{j} O_{\mu_{j}} S_{\mu_{j}} \subset W$ for some $1 \leq j \leq n$.

It is easy to verify that $D(\mathcal{U})$ equipped with this topology is a Hausdorff space, and that both $A$ and $\mathcal{N}(\mathcal{U})$, as subspaces of $D(\mathcal{U})$, preserve their original topologies. Moreover, $\mathcal{N}(\mathcal{U})$ is open in $D(\mathcal{U})$, and hence $A$ is closed in $D(\mathcal{U})$. We equip $D(\mathcal{U})$ with the natural action of $G$ in such a way that $A$ and $N(\mathcal{U})$ are invariant subsets of $D(\mathcal{U})$, and we call the resulting $G$-space $D(\mathcal{U})$ the Dugundji $G$-replacement (associated with the $G$-normal cover $\mathcal{U}$ ).

Lemma 3.3. The natural action of $G$ on $D(\mathcal{U})$ is continuous.

Proof. One only needs to check continuity at each $a \in A$. Let $g \in G$ and $\widetilde{V}$ be a basic neighborhood of $g a$ in $D(\mathcal{U})$. By continuity of the $G$-action on $Y$, there are a neighborhood $W$ of $a$ in $Y$ and a neighborhood $O$ of $g$ in $G$ such that $O \cdot W \subset V$. One easily sees that $O \cdot \widetilde{W} \subset \widetilde{V}$, completing the proof.

Lemma 3.4. Let $Y$ be a $G$-space, $A$ a closed invariant subset of $Y$, and $\mathcal{U}=\left\{g O_{\mu} S_{\mu} \mid g \in G, \mu \in \mathcal{M}\right\}$ a Dugundji $G$-cover of $Y \backslash A$ with large slicing subgroups $\left\{H_{\mu} \mid \mu \in \mathcal{M}\right\}$. Then there exists a $G$-map $q: Y \rightarrow D(\mathcal{U})$ such that

(1) $\left.q\right|_{A}$ is the identical homeomorphism,

(2) $q(Y \backslash A) \subset D(\mathcal{U}) \backslash q(A)$.

Proof. Choosing an invariant partition of unity subordinated to the invariant cover $\left\{G\left(S_{\mu}\right) \mid \mu \in \mathcal{M}\right\}$, one can define a $G$-map $p: Y \backslash A \rightarrow \mathcal{N}(\mathcal{U})$ as in Lemma 3.2. Now we define $q: Y \rightarrow D(\mathcal{U})$ by setting

$$
q(y)= \begin{cases}y & \text { if } y \in A \\ p(y) & \text { if } y \in Y \backslash A .\end{cases}
$$


Clearly, (1) and (2) hold. Let us check that $q$ is continuous. For the continuity on $Y \backslash A$ we refer to Lemma 3.2. To prove the continuity on $A$, fix $a \in A$ and let $\widetilde{V}$ be a basic neighborhood of $q(a)$ in $D(\mathcal{U})$. By Definition 2.5, there exists a neighborhood $W \subset V$ of $a$ in $Y$ such that every $g O_{\mu} S_{\mu}$ which meets $W$ is contained in $V$.

We claim that $q(W) \subset \widetilde{V}$. Indeed, if $y \in A \cap W$ then $q(y)=y \in A \cap W \subset$ $A \cap V \subset \widetilde{V}$. Now let $y \in(Y \backslash A) \cap W$, and let $\mu_{1}, \ldots, \mu_{n}$ be all the indices such that $y \in g_{\mu_{1}} S_{\mu_{1}} \cap \cdots \cap g_{\mu_{n}} S_{\mu_{n}}$ for some $g_{\mu_{1}}, \ldots, g_{\mu_{n}} \in G$. Then each $g_{\mu_{i}} S_{\mu_{i}}, 1 \leq i \leq n$, meets $W$ at $y$, and hence

$$
g_{\mu_{1}} O_{\mu_{1}} S_{\mu_{1}} \cup \cdots \cup g_{\mu_{n}} O_{\mu_{n}} S_{\mu_{n}} \subset V,
$$

so that $\left\langle g_{\mu_{1}} p_{\mu_{1}} H_{\mu_{1}}, \ldots, g_{\mu_{n}} p_{\mu_{n}} H_{\mu_{n}}\right\rangle \subset \widetilde{V}$ for all $p_{\mu_{i}} \in O_{\mu_{i}}$. Since $q(y)=$ $p(y) \in\left\langle g_{\mu_{1}} p_{\mu_{1}} H_{\mu_{1}}, \ldots, g_{\mu_{n}} p_{\mu_{n}} H_{\mu_{n}}\right\rangle$, we see that $q(y) \in \widetilde{V}$. The continuity of $q$ is proved. Its equivariance follows from the invariance of $\varphi_{\mu}$ and the equivariance of $f_{\mu}, \mu \in \mathcal{M}$.

LEMma 3.5. Under the hypotheses of Lemma 3.3, for every neighborhood $U$ of $A$ in $D(\mathcal{U})$, there exists an invariant neighborhood $V$ of $A$ in $D(\mathcal{U})$ such that $\bar{V} \subset U$.

Proof. One can assume that $U=\bigcup_{a \in A} \widetilde{U}_{a}$, where $U_{a}$ is a neighborhood of $a$ in $Y$, and $U_{g a}=g U_{a}$ for all $g \in G$ and $a \in A$.

By Definition 2.5, choose a neighborhood $V_{a} \subset U_{a}$ of $a$ in $Y$ such that every $g O_{\mu} S_{\mu} \in \mathcal{U}$ which meets $V_{a}$ is contained in $U_{a}$. Clearly, one can assume that $V_{g a}=g V_{a}$ for all $g \in G$ and $a \in A$.

We claim that $V=\bigcup_{a \in A} \widetilde{V}_{a}$ is the desired neighborhood of $A$. Indeed, for every $G$-vertex $G / H_{\mu}$, we define the open star by

$$
\operatorname{St}\left(G / H_{\mu}, \mathcal{N}(\mathcal{U})\right)=\left\{\sum_{\lambda \in \mathcal{M}} t_{\lambda} g_{\lambda} H_{\lambda} \in \mathcal{N}(\mathcal{U}) \mid t_{\mu}>0\right\} .
$$

It is not difficult to find that $V$ is the union of $A$ and all the open stars $\operatorname{St}\left(G / H_{\mu}, \mathcal{N}(\mathcal{U})\right)$ for which $O_{\mu} S_{\mu} \subset V_{a}$ for some $a \in A$. Then the closure $\bar{V}$ is just the union of $A$ and all the corresponding closed stars

$$
\overline{\operatorname{St}}\left(G / H_{\mu}, \mathcal{N}(\mathcal{U})\right)=\left\{\sum_{\lambda \in \mathcal{M}} t_{\lambda} g_{\lambda} H_{\lambda} \in \mathcal{N}(\mathcal{U}) \mid t_{\mu} \geq 0\right\} .
$$

Now let $\left\langle g_{\mu_{0}} p_{\mu_{0}} H_{\mu_{0}}, \ldots, g_{\mu_{n}} g_{\mu_{n}} H_{\mu_{n}}\right\rangle$ be any closed cell contained in the closed star $\overline{\operatorname{St}}\left(G / H_{\mu_{0}}, \mathcal{N}(\mathcal{U})\right)$ with $O_{\mu_{0}} S_{\mu_{0}} \subset V_{a}$ for some $a \in A$. It suffices to show that $\left\langle g_{\mu_{0}} p_{\mu_{0}} H_{\mu_{0}}, \ldots, g_{\mu_{n}} g_{\mu_{n}} H_{\mu_{n}}\right\rangle$ is contained in $U$.

By the definition of the cell $\left\langle g_{\mu_{0}} p_{\mu_{0}} H_{\mu_{0}}, \ldots, g_{\mu_{n}} g_{\mu_{n}} H_{\mu_{n}}\right\rangle$, there exists a point $x \in \bigcap_{i=0}^{n} G\left(S_{\mu_{i}}\right)$ such that $f_{\mu_{i}}(x)=g_{\mu_{i}} H_{\mu_{i}}$ for all $0 \leq i \leq n$, where $f_{\mu_{i}}: G\left(S_{\mu_{i}}\right) \rightarrow G / H_{\mu_{i}}$ is the slicing map (see Section 2). This shows, in turn, that $x \in \bigcap_{i=0}^{n} g_{\mu_{i}} S_{\mu_{i}}$, and in particular, $\bigcap_{i=0}^{n} g_{\mu_{i}} O_{\mu_{i}} S_{\mu_{i}} \neq \emptyset$. 
But remember that $O_{\mu_{0}} S_{\mu_{0}} \subset V_{a}$ for some $a \in A$, so $g_{\mu_{0}} O_{\mu_{0}} S_{\mu_{0}} \subset$ $g_{\mu_{0}} V_{a}=V_{g_{\mu_{0}} a}$. Now, due to the choice of the neighborhood $V_{g_{\mu_{0}} a}$, each $g_{\mu_{i}} O_{\mu_{i}} S_{\mu_{i}}, 0 \leq i \leq n$, is contained in $U_{a}$ because $g_{\mu_{i}} O_{\mu_{i}} S_{\mu_{i}} \cap V_{g_{\mu_{0}} a} \supset$ $g_{\mu_{i}} O_{\mu_{i}} S_{\mu_{i}} \cap g_{\mu_{0}} O_{\mu_{0}} S_{\mu_{0}} \neq \emptyset$. This shows that the closed cell $\left\langle g_{\mu_{0}} p_{\mu_{0}} H_{\mu_{0}}, \ldots\right.$ $\left.\ldots, g_{\mu_{n}} g_{\mu_{n}} H_{\mu_{n}}\right\rangle$ is contained in $\widetilde{U}_{a}$, and hence in $U$, as required.

\section{Proof of Main Theorem}

Lemma 4.1. Let $(Y, A)$ be a $G$-pair, $\mathcal{U}=\left\{g O_{\mu} S_{\mu} \mid g \in G, \mu \in \mathcal{M}\right\}$ a Dugundji $G$-cover of $Y \backslash A$ with large slicing subgroups $\left\{H_{\mu} \mid \mu \in \mathcal{M}\right\}$, and let $X$ be a $G$-ANR. Then every $G$-map $\varphi: A \rightarrow X$ admits a $G$-extension $\varphi^{\prime}: U \rightarrow X$ over a neighborhood $U$ of $A$ in the Dugundji G-replacement $D(\mathcal{U})$.

Proof. One can assume that $X$ is a closed invariant subset of a $G$-space of the form $C(G, L)$, where $L$ is a normed linear space and $C(G, L)$ is the normed linear $G$-space of all continuous maps $G \rightarrow L$, endowed with the sup-norm and the linear $G$-action defined by the formula $(g f)(x)=f(x g)$ for $g, x \in G, f \in C(G, L)$ (see [2, Theorem 3]).

Let $p: C(G, L) \rightarrow L$ be the evaluation map defined by $p(f)=f(e)$, where $f \in C(G, L)$ and $e$ is the unity of $G$. Clearly $p$ is continuous. Set $f=p \varphi$.

First we shall extend $f$ to a continuous map $F: D(\mathcal{U}) \rightarrow L$. Then the map $f^{\prime}: D(\mathcal{U}) \rightarrow C(G, L)$ defined by $f^{\prime}(z)(g)=F(g z), z \in Z, g \in G$, is a $G$-extension of $f$. Further, since $X$ is a $G$-ANR, there exists a $G$-retraction $r: T \rightarrow X$ for some invariant neighborhood $T$ of $X$ in $C(G, L)$. Put $U=$ $\left(f^{\prime}\right)^{-1}(T)$ and $\varphi^{\prime}=r f^{\prime}$. Then the map $\varphi^{\prime}: U \rightarrow X$ is the desired $G$-extension of $\varphi$.

Now, let us proceed with the construction of $F: D(\mathcal{U}) \rightarrow L$.

Let $\mathcal{N}_{k}(\mathcal{U})$ denote the $G$ - $k$-skeleton of $\mathcal{N}(\mathcal{U})$, i.e., $\mathcal{N}_{k}(\mathcal{U})$ is the union of all $G$ - $m$-simplices of $\mathcal{N}(\mathcal{U})$ with $m \leq k$.

First we extend $f$ over each $G$-vertex $G / H_{\mu} \subset \mathcal{N}_{0}(\mathcal{U})$. To this end, for every $G$-vertex $G / H_{\mu}$ choose a finite open cover of the form

$$
\left\{g_{1} O_{\mu} H_{\mu}, \ldots, g_{n} O_{\mu} H_{\mu}\right\}
$$

where $n$ and $g_{1}, \ldots, g_{n}$ depend upon $\mu \in \mathcal{M}$. Of course, $g_{k}$ is not uniquely determined by $g_{k} O_{\mu} H_{\mu}$, but for our further constructions it suffices to fix just one such element. Let $\left\{\varphi_{1}, \ldots, \varphi_{n}\right\}$ be a partition of unity of $G / H_{\mu}$ subordinated to this cover, i.e., $\varphi_{k}^{-1}((0,1]) \subset g_{k} O_{\mu} H_{\mu}, 1 \leq k \leq n$. For each $\mu \in \mathcal{M}$ choose an $x_{\mu} \in S_{\mu}$ and associate to it a point $a_{\mu} \in A$ such that $\varrho\left(x_{\mu}, a_{\mu}\right)<2 \varrho\left(x_{\mu}, A\right)$. Since $A$ is an invariant subset and $\varrho$ is an invariant metric,

$$
\varrho\left(g x_{\mu}, g a_{\mu}\right)<2 \varrho\left(g x_{\mu}, A\right) \quad \text { for all } g \in G \text { and } \mu \in \mathcal{M} \text {. }
$$


Define $\Phi: A \cup \mathcal{N}_{0}(\mathcal{U}) \rightarrow L$ by setting

$$
\Phi(x)= \begin{cases}f(x), & \text { if } x \in A, \\ \varphi_{1}(x) f\left(g_{1} a_{\mu}\right)+\cdots+\varphi_{n}(x) f\left(g_{n} a_{\mu}\right) & \text { if } x \in G / H_{\mu} .\end{cases}
$$

As $f\left(g_{1} a_{\mu}\right), \ldots, f\left(g_{n} a_{\mu}\right)$ do not depend on $x \in G / H_{\mu}$, and $\varphi_{1}, \ldots, \varphi_{n}$ are continuous functions on $G / H_{\mu}$, we conclude that $\Phi$ is continuous at each point of $G / H_{\mu}$. Consequently, $\Phi$ is continuous on $\mathcal{N}_{0}(\mathcal{U})$.

Let us verify continuity of $\Phi$ at each $a \in A$. Let $M$ be a neighborhood of $\Phi(a)=f(a)$ in $L$. Of course, one can assume that $M$ is an open ball centered at $f(a)$. By continuity of $f$, there exists a $\delta>0$ such that $f(N(a, \delta) \cap A) \subset M$. Recall that $N(y, r)$ denotes the open ball in $Y$ of radius $r$ centered at $y \in Y$.

Let $V=N(a, \delta / 3)$. By Definition 2.5, there exists a neighborhood $W \subset$ $V$ of $a$ in $Y$ such that every $g O_{\mu} S_{\mu}$ that meets $W$ is contained in $V$. We claim that $\Phi\left(\widetilde{W} \cap\left(A \cup \mathcal{N}_{0}(\mathcal{U})\right)\right) \subset M$, where $\widetilde{W}$ is the basic neighborhood of $a$ in $D(\mathcal{U})$ defined by $W$ (see Section 3, the paragraph after Lemma 3.2).

Indeed, $\widetilde{W} \cap A=W \cap A \subset N(a, \delta) \cap A$, so $f(\widetilde{W} \cap A) \subset M$.

If $x \in \widetilde{W} \cap \mathcal{N}_{0}(\mathcal{U})$ then $x=g_{1} H_{\mu} \in G / H_{\mu}$ for some $G$-vertex $G / H_{\mu}$ and $g_{1} O_{\mu} S_{\mu} \subset W$, where $g_{1} \in G$. Let $g_{1} O_{\mu} H_{\mu}, \ldots, g_{k} O_{\mu} H_{\mu}$ be all the elements of the cover $\left\{g_{1} O_{\mu} H_{\mu}, \ldots, g_{n} O_{\mu} H_{\mu}\right\}$ which contain $x$. Then $\bigcap_{i=1}^{k} g_{i} O_{\mu} S_{\mu} \neq \emptyset$.

Since $g_{1} O_{\mu} S_{\mu} \subset W$, we infer that $g_{i} O_{\mu} S_{\mu} \cap W \neq \emptyset, 1 \leq i \leq k$. Hence, due to the choice of $W$, each $g_{i} O_{\mu} S_{\mu}$ must be contained in $V$.

Therefore, $\varrho\left(g_{i} x_{\mu}, a\right)<\delta / 3$ for all $i=1, \ldots, k$. Using (4.1), we get

$$
\begin{aligned}
\varrho\left(g_{i} a_{\mu}, a\right) & \leq \varrho\left(g_{i} a_{\mu}, g_{i} x_{\mu}\right)+\varrho\left(g_{i} x_{\mu}, a\right)<2 \varrho\left(g_{i} x_{\mu}, A\right)+\varrho\left(g_{i} x_{\mu}, a\right) \\
& \leq 2 \varrho\left(g_{i} x_{\mu}, a\right)+\varrho\left(g_{i} x_{\mu}, a\right)<2 \delta / 3+\delta / 3=\delta .
\end{aligned}
$$

Hence all the points $g_{i} a_{\mu}, i=1, \ldots, k$, belong to the ball $N(a, \delta)$, which yields $f\left(g_{1} a_{\mu}\right), \ldots, f\left(g_{n} a_{\mu}\right) \in M$. Since $M$ is convex, it then follows from the definition of $\Phi(x)$ that $\Phi(x) \in M$. Thus, $\Phi$ is continuous on $A \cup \mathcal{N}_{0}(\mathcal{U})$.

Since $L$ is a linear space, we can extend $\Phi$ linearly over each $G$-simplex of $\mathcal{N}(\mathcal{U})$ to obtain a map $F: D(\mathcal{U}) \rightarrow X$. More precisely, if $\sigma=\left\langle\mu_{0}, \ldots, \mu_{n}\right\rangle$ is a simplex and $\sum_{i=0}^{n} t_{\mu_{i}} g_{\mu_{i}} p_{\mu_{i}} H_{\mu_{i}}$ is a point of the $G$-simplex $\Delta(\sigma) \subset \mathcal{N}(\mathcal{U})$, then we set

$$
F\left(\sum_{i=0}^{n} t_{\mu_{i}} g_{\mu_{i}} p_{\mu_{i}} H_{\mu_{i}}\right)=\sum_{i=0}^{n} t_{\mu_{i}} \Phi\left(g_{\mu_{i}} p_{\mu_{i}} H_{\mu_{i}}\right) .
$$

Clearly, $F$ is continuous on each $G$-simplex of $\mathcal{N}(\mathcal{U})$. Since $\mathcal{N}(\mathcal{U})$ is endowed with the weak topology defined by its closed $G$-simplices, $F$ is continuous on $\mathcal{N}(\mathcal{U})$. It remains to check the continuity on $A$.

Fix $a \in A$ and let $M$ be a neighborhood of $F(a)=\Phi(a)$ in $L$. Clearly, one can assume that $M$ is an open ball centered at $f(a)$. 
By continuity of $\Phi$, there exists a basic neighborhood $\widetilde{V}$ of $a$ in $\mathcal{N}(\mathcal{U})$ such that $\Phi\left(\widetilde{V} \cap\left(A \cup \mathcal{N}_{0}(\mathcal{U})\right)\right) \subset M$, where $V$ is a neighborhood of $a$ in $Y$. By Definition 2.5, there exists a neighborhood $W \subset V$ of $a$ in $Y$ such that every $g O_{\mu} S_{\mu}$ which meets $W$ is contained in $V$. We claim that $F(\widetilde{W}) \subset M$.

Indeed, $\widetilde{W} \cap A=W \cap A \subset V \cap A$, so $F(\widetilde{W} \cap A)=\Phi(W \cap A) \subset \Phi(V \cap A) \subset M$. If $x \in \widetilde{W} \cap \mathcal{N}(\mathcal{U})$ then $x$ belongs to an open cell

$$
\left(g_{\mu_{0}} p_{\mu_{1}} H_{\mu_{1}}, \ldots, g_{\mu_{n}} p_{n} H_{\mu_{n}}\right) \subset \widetilde{W} \cap \mathcal{N}(\mathcal{U})
$$

with $g_{\mu_{i}} \in G, p_{\mu_{i}} \in O_{\mu_{i}}, 0 \leq i \leq n$, such that $g_{\mu_{j}} O_{\mu_{j}} S_{\mu_{j}} \subset W$ for some $0 \leq j \leq n$.

It follows from the definition of $\left(g_{\mu_{0}} p_{\mu_{0}} H_{\mu_{0}}, \ldots, g_{\mu_{n}} p_{n} H_{\mu_{n}}\right)$ that $\bigcap_{i=0}^{n} g_{\mu_{i}} O_{\mu_{i}} S_{\mu_{i}} \neq \emptyset$ (cf. the proof of Lemma 3.5). Since $g_{\mu_{j}} O_{\mu_{j}} S_{\mu_{j}} \subset W$, it follows that every $g_{\mu_{i}} O_{\mu_{i}} S_{\mu_{i}}$ meets $W$, and is contained in $V$. Consequently, each cell vertex $g_{\mu_{i}} p_{\mu_{i}} H_{\mu_{i}}$ belongs to $\widetilde{V} \cap \mathcal{N}_{0}(\omega)$, and so $\Phi\left(g_{\mu_{i}} p_{\mu_{i}} H_{\mu_{i}}\right) \in M$. But $F(x)$ is a convex combination of the points $\Phi\left(g_{\mu_{i}} p_{\mu_{i}} H_{\mu_{i}}\right), 0 \leq i \leq n$, and since $M$ is convex, we conclude that $F(x) \in M$.

Proof of Main Theorem. The "only if" part is evident. To prove the "if" part, we first observe that the $H$-fixed point sets of a $G$-ANR are ANR's [1, Theorem 7]. On the other hand, it is well known that a homotopy trivial ANR is an AR [14, Theorem 4.2.20]. So, in our case each $X^{H}$ is an AR. Using this fact, let us prove that $X$ is a $G$-AR.

Indeed, let $Y$ be a metrizable $G$-space, $A$ a closed invariant subset of $Y$, and $\varphi: A \rightarrow X$ a $G$-map. We shall show that $\varphi$ extends to a $G$-map $\Phi: Y \rightarrow X$.

Choose a Dugundji $G$-cover $\mathcal{U}=\left\{g O_{\mu} S_{\mu} \mid g \in G, \mu \in \mathcal{M}\right\}$ of $Y \backslash A$ with large slicing subgroups $\left\{H_{\mu} \mid \mu \in \mathcal{M}\right\}$ (see Lemma 2.6). Let $\mathcal{N}(\mathcal{U})$ denote the $G$-nerve of $\mathcal{U}$ and let $q: Y \rightarrow D(\mathcal{U})$ be a $G$-map as in Lemma 3.4. It is sufficient to prove that $\varphi: A \rightarrow X$ extends to a $G$-map $F: D(\mathcal{U}) \rightarrow X$. Then $\Phi=F q$ is as desired.

Let $\mathcal{N}_{k}(\mathcal{U})$ denote the $G$ - $k$-skeleton of $\mathcal{N}(\mathcal{U})$. Since $X$ is a $G$-ANR, by Lemma 4.1, $\varphi$ extends to a $G$-map $\varphi^{\prime}: U \rightarrow X$, where $U$ is a $G$-invariant neighborhood of $A$ in $D(\mathcal{U})$. By Lemma 3.5, choose a $G$-invariant neighborhood $V$ of $A$ in $D(\mathcal{U})$ such that $\bar{V} \subset U$.

We will extend $\varphi$, by induction on the dimension $k$, to a $G$-map $f_{k}: \bar{V} \cup$ $\mathcal{N}_{k}(\mathcal{U}) \rightarrow X, k \geq 0$, and thus obtain the desired $G$-extension $F: D(\mathcal{U}) \rightarrow X$.

First we define $f_{0}: \bar{V} \cup \mathcal{N}_{0}(\mathcal{U}) \rightarrow X$. Since $X^{G} \neq \emptyset$, we choose $v \in X^{G}$. If $G / H_{\mu}$ is a $G$-vertex in $\mathcal{N}_{0}(\mathcal{U}) \backslash \bar{V}$, we set $f_{0}\left(g H_{\mu}\right)=v, g H_{\mu} \in G / H_{\mu}$. We also put $\left.f_{0}\right|_{\bar{V}}=\left.\varphi^{\prime}\right|_{\bar{V}}$. Since $f_{0}$ is continuous at each $G$-vertex $G / H_{\mu}$, it is continuous on $\mathcal{N}_{0}(\mathcal{U})$. Since it coincides with $\varphi^{\prime}$ on $V$, it is continuous on $A$ as well. Moreover, it preserves the action of $G$, and hence it is a $G$-extension of $\varphi$. 
Now suppose a $G$-extension $f_{k}: \bar{V} \cup \mathcal{N}_{k}(\mathcal{U}) \rightarrow X$ of $f_{k-1}, k \geq 1$, is already constructed. We shall construct a $G$-extension $f_{k+1}: \bar{V} \cup \mathcal{N}_{k+1}(\mathcal{U}) \rightarrow X$ of $f_{k}$.

Let $\sigma=\left\langle\mu_{0}, \ldots, \mu_{k+1}\right\rangle$ be a $k+1$-simplex in $\widetilde{\mathcal{N}}(\mathcal{U})$ such that the $G$ - $(k+1)$ simplex $\Delta(\sigma) \subset \mathcal{N}_{k+1}(\mathcal{U})$ is not contained in $\bar{V}$. Consider the following closed $G$-invariant subset of $\Delta(\sigma)$ :

$$
B=(\bar{V} \cap \Delta(\sigma)) \cup \partial \Delta(\sigma),
$$

where $\partial \Delta(\sigma)$ is the $G$-boundary of $\Delta(\sigma)$. Observe that $f_{k}$ is defined on $B$ because $\bar{V} \cap \Delta(\sigma) \subset \bar{V}$ and $\partial \Delta(\sigma) \subset \mathcal{N}_{k}(\mathcal{U})$.

Let $N_{\mu_{i}}, 0 \leq i \leq k+1$, be a closed normal subgroup of $G$ such that $N_{\mu_{i}} \subset H_{\mu_{i}}$ and $G / N_{\mu_{i}}$ is a Lie group. Put $N=\bigcap_{i=0}^{k+1} N_{\mu_{i}}$. This is clearly a closed normal subgroup of $G$ and the quotient group $G / N$ is a Lie group (see e.g. [19, Ch. 8, Section 46 (A)]). It is clear that $N \subset G_{b} \subset G_{f_{k}(b)}$ for every $b \in B$. In other words, $f_{k}$ maps $B$ into the $N$-fixed point set $X^{N}$.

Since $N$ is a normal subgroup of $G, X^{N}$ is a $G$-invariant subset of $X$, and hence, a $G$-space. As $N$ acts trivially on the join $\mathcal{J}(\sigma)=G / H_{\mu_{0}}$ * $\cdots * G / H_{\mu_{k+1}}$ and on $X^{N}$, we can consider these $G$-spaces as $G / N$-spaces endowed with the induced $G / N$-action.

Now we aim at extending the $G / N$-map $f_{k}: B \rightarrow X^{N}$ to a $G / N$-map $f_{\sigma}: \Delta(\sigma) \rightarrow X^{N}$. Observe that for any closed subgroup $K^{\prime} \subset G / N$, the set $\left(X^{N}\right)^{K^{\prime}}$ of $K^{\prime}$-fixed points in $X^{N}$ is an AR. Indeed, let $K$ be the preimage of $K^{\prime}$ under the natural homomorphism $G \rightarrow G / N$. Then it is clear that $\left(X^{N}\right)^{K^{\prime}}=X^{K}$. But $X^{K}$ is an AR by the hypothesis.

Claim. The join $\mathcal{J}(\sigma)$ has finitely many $G / N$-orbit types.

Proof. First we observe that $G / H_{\mu_{i}}$ is naturally $G / N$-homeomorphic to $(G / N) /\left(H_{\mu_{i}} / N\right)$. Since $G / N$ is a Lie group, by [17, Proposition 1.4.1], $G / H_{\mu_{i}}$ can be $G / N$-equivariantly embedded into a Euclidean $G / N$-space $E_{i}$ equipped with an orthogonal action of $G / N$. This gives rise to a natural $G / N$-equivariant embedding of $\mathcal{J}(\sigma)$ into $E=E_{0} \oplus \cdots \oplus E_{k+1}$ endowed with the diagonal action of $G / N$. Since $E$ is a Euclidean $G / N$-space, it has finitely many $G / N$-orbit types (see [17, Corollary 1.7.26]). This implies that $\mathcal{J}(\sigma)$ also has finitely many $G / N$-orbit types, proving the claim.

Further, since $\Delta(\sigma)$ is an invariant subset of $\mathcal{J}(\sigma)$, which in turn is a subset of a Euclidean $G / N$-space (see the proof of the Claim), we infer that $\Delta(\sigma)$ is a finite-dimensional, separable, metrizable $G / N$-space with finitely many $G / N$-orbit types. Thus, the hypotheses of Jaworowski-Lashof Theorem 2.7 are fulfilled, and so $\left.f_{k}\right|_{B}$ admits a $G / N$-extension $f_{\sigma}: \Delta(\sigma) \rightarrow X^{N}$.

In what follows we will consider $f_{\sigma}$ as a $G$-map from $\Delta(\sigma)$ to $X$. 
Next, we define the map $f_{k+1}: \bar{V} \cup \mathcal{N}_{k+1}(\mathcal{U}) \rightarrow X$ by setting

$$
f_{k+1}(z)= \begin{cases}f_{\sigma}(z) & \text { if } z \in \Delta(\sigma) \text { with } \operatorname{dim} \sigma=k+1, \\ f_{k}(a) & \text { if } a \in \bar{V} \cup \mathcal{N}_{k}(\mathcal{U}) .\end{cases}
$$

Then $f_{k+1}$ is well defined and extends $f_{k}$. Since $\left.f_{k+1}\right|_{\Delta(\tau)}$ is continuous for every $G$-simplex $\Delta(\tau) \subset \mathcal{N}_{k+1}(\mathcal{U})$, we see that $f_{k+1}$ is continuous on $\mathcal{N}_{k+1}(\mathcal{U})$. As $f_{k+1}$ coincides with $\varphi^{\prime}$ on $V$, it is continuous at the points of $A$ as well. It is also clear that $f_{k+1}$ preserves the action of $G$. This completes the inductive step.

Now, we define the desired $G$-map $F: D(\mathcal{U}) \rightarrow X$ by setting

$$
F(z)=f_{k}(z) \quad \text { whenever } \quad z \in A \cup \mathcal{N}_{k}(\mathcal{U}), k \geq 0 .
$$

Clearly, $F$ is well defined and preserves the $G$-action. Its continuity on $\mathcal{N}(\mathcal{U})$ follows from the continuity of $\left.F\right|_{\Delta(\tau)}$ for each $G$-simplex $\Delta(\tau) \subset \mathcal{N}(\mathcal{U})$. Since $F$ coincides with $\varphi^{\prime}$ on the neighborhood $V$ of $A$, it is continuous on $A$ as well. This completes the proof of the Main Theorem.

\section{References}

[1] S. A. Antonyan, Retracts in categories of G-spaces, Izv. Akad. Nauk Arm. SSSR Ser. Mat. 15 (1980), 365-378 (in Russian); English transl.: Soviet J. Contemp. Math. Anal. 15 (1980), 30-43.

[2] -, Equivariant embeddings into $G$-AR's, Glas. Mat. 22 (42) (1987), 503-533.

[3] - Existence of a slice for arbitrary compact transformation groups, Mat. Zametki 56 (1994), no. 5, 3-9 (in Russian); English transl.: Math. Notes 56 (1994), 1101-1104.

[4] -, Universal proper G-spaces, Topology Appl. 117 (2002), 23-43.

[5] - West's problem on equivariant hyperspaces and Banach-Mazur compacta, Trans. Amer. Math. Soc. 355 (2003), 3379-3404.

[6] -, Orbit spaces and unions of equivariant absolute neighborhood extensors, Topology Appl. 146-147 (2005), 289-315.

[7] G. Bredon, Introduction to Compact Transformation Groups, Academic Press, New York, 1972.

[8] J. Dugundji, An extension of Tietze's theorem, Pacific J. Math. 1 (1951), 353-367.

[9] - Topology, Allyn and Bacon, Boston, 1966.

[10] I. M. James and G. B. Segal, On equivariant homotopy theory, in: Lecture Notes in Math. 788, Springer, 1980, 316-330.

[11] J. Jaworowski, Extension of G-maps and Euclidean G-retracts, Math. Z. 146 (1976), $143-148$.

[12] V. L. Klee, A note on topological properties of normed linear spaces, Proc. Amer. Math. Soc. 7 (1956), 673-674.

[13] R. Lashof, The equivariant extension theorem, Proc. Amer. Math. Soc. 83 (1981), $138-140$.

[14] J. van Mill, The Infinite-Dimensional Topology of Function Spaces, Elsevier, Amsterdam, 2001.

[15] J. Milnor, Construction of universal bundles, II, Ann. of Math. 63 (1956), 430-436.

[16] G. D. Mostow, Equivariant embeddings in Euclidean space, ibid. 65 (1957), 432-446. 
[17] R. Palais, The classification of G-spaces, Mem. Amer. Math. Soc. 36 (1960).

[18] -, On the existence of slices for actions of noncompact Lie groups, Ann. of Math. 73 (1961), 295-323.

[19] L. S. Pontryagin, Selected Works. Volume 2. Topological Groups, Classics of Soviet Math., Gordon \& Breach, New York, 1986.

[20] J. E. West, Infinite products which are Hilbert cubes, Trans. Amer. Math. Soc. 150 (1970), 1-25.

Departamento de Matemáticas

Facultad de Ciencias

Universidad Nacional Autónoma de México

04510, México, D.F., México

E-mail: antonyan@servidor.unam.mx

Received 15 September 2005;

in revised form 22 September 2007 